\title{
MATEMATICAL MODEL FOR YARN UNWINDING FROM PACKAGES
}

\author{
Stanislav Praček,Sluga Franci \\ Department of textile, NTF, University of Ljubljana, Snežniška 5, \\ SI-1000 Ljubljana, Slovenija, stane.pracek@ntf.uni-lj.si
}

\begin{abstract}
Yarn unwinding from a package is important in many textile processes. The stability of the unwinding process has a direct influence on the efficiency of the process and on the quality of the end product. During the unwinding, the tension is oscillating. This is especially noticeable in over-end unwinding from a static package, where the yarn is being withdrawn with a high velocity in the direction of the package axis. The optimal form of the package allows an optimal shape of the yarn balloon and low and steady tension even at very high unwinding velocities. The purpose of this work is to write down the equations that describe the motion of yarn during unwinding and to construct a mathematical model whichwould permit to simulate the process of unwinding.
\end{abstract}

Keywords- yarn unwinding, balloon theory, simulations

\section{INTRODUCTION}

Yarn unwinding is an essential step in many textile processes [1]. The quality of the fabric that is produced directly depends on the regularity of the unwinding: the tension in the yarn should be low and constant. The characteristics of the unwinding process are thus important for production of high quality garments and should therefore be optimized.

The teory of unwinding and balloon formation originate from the pioneering work of D. Padfield[2]. She modified Mack's equations[3] and included terms that describe the Corolis force. She found solution for a balloon that forms during unwinding from stationary cylindrical package when quasistationary conditions apply. This theory was also used for calculations of multiple balloons and for balloons formed during unwinding from packages with different geometry, such as conic packages[4]. Kothari and Leaf have rederived similar equations, additionally taking into account the effect of gravity and tangential air drag[5]. Using elaborate numerical calculates they have shown that these two forces have only a small contribution to overall dynamics of the yarn. Recently Fraser et al. have applied mathematical theory of perturbations to correctly eliminate the time dependance from equations of motion in stationary conditions[6]. They have show that the entire time dependance can be shifted to moving boundary conditions. In this manner the innitial-value problem of partial differential equations can be reduced to a boundary problem which is much easier to solve.

Bulding on this foundation we have simplified the problem even further. We will show how a simple model function that describes the package can be used to estimate the unwinding properties of packages of different geometries and different winding types. 


\section{THEORETICAL PART}

The yarn is being withdrawn with velocity $\mathrm{V}$ through an eyelet, where we also fix the origin $\mathrm{O}$ of our coordinate system (Fig.1). The yarn is rotating aroun the $\mathrm{z}$ axis with an angular velocity $\omega$. At the lift-off point Dv the yarn lifts from the package and forms a balloon. At the unwinding point Od the yarn starts to slide on the surface of the package. Angle $\phi$ is the winding angle of the yarn on the package.

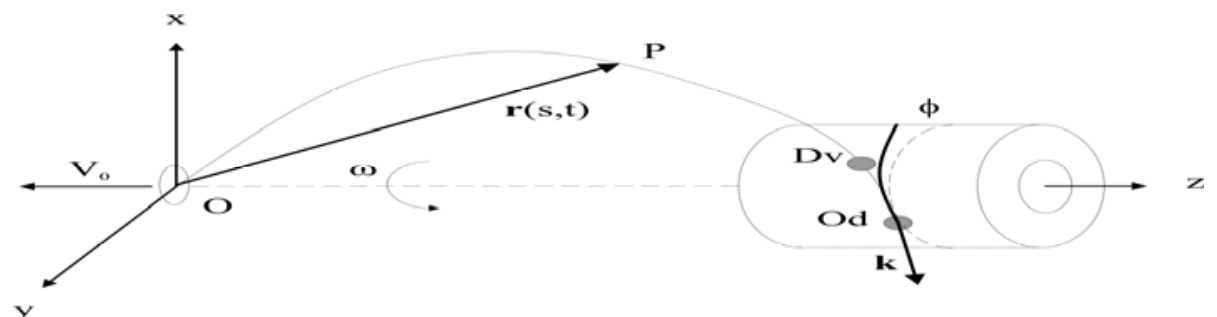

Fig.1 Mechanical setup in overend yarn unvinding from cilimdrical package

We now consider the equation of motion of yarn [7]:

$$
\rho\left(D^{2} r+2 \omega \times D r+\omega \times(\omega \times r)+\omega \times r\right)=\frac{\partial}{\partial s}\left(T \frac{\partial r}{\partial s}\right)+f
$$

Here $\rho$ is the mass per unit length of the yarn, $\mathbf{r}$ is the radius vector to a given point on the yarn, $\mathrm{D}$ is the comoving time derivative operator, $\mathrm{T}$ is the yarn tension and $\mathbf{f}$ is the density of external forces acting on the yarn. In the part of the yarn that forms the balloon $\mathbf{f}$ is the density of the air drag force[8]:

$$
f=-\frac{1}{2} c_{u} \rho d\left|v_{n}\right| v_{n}
$$

where $c_{u}$ is the air drag coefficient, $d$ is the diameter of the yarn and $v_{n}$ is the normal component of the yarn velocity. In the part of the yarn that slides on the package (between the unwinding and lift-off point) $\mathrm{f}$ is the density of forces with which package resists the motion of the yarn. It consists of the normal force of the package and the force of friction:

$$
f=n e_{r}-\mu n \frac{v}{|v|}
$$

where $\mathrm{n}$ is the strength of normal force, $e_{r}$ is the radial unit vector and $\mu$ is the coefficient of friction.It is worthwhile to cast the equation of motion in the dimensionless form. This consists of finding "natural" units in which to express all the quantities appearing in the equation. A number of constants will remain in the reduced equation and these dimensionless parameters have the most pronounced effect on the yarn dynamics.

We express all distances in units of package radius:

$$
\bar{r}=\frac{r}{C}, \bar{Z}=\frac{Z}{C}, \bar{S}=\frac{S}{C}
$$

time is expressed in units of period of balloon rotation:

$$
\bar{t}=\frac{t}{\tau}=\omega t
$$


velocitis are expressed in units of unwinding speeed:

$$
\bar{v}=\frac{v}{V}, \bar{v}_{n}=\frac{v_{n}}{V}
$$

and finally we find the following suitable combinations of quantities for forces and tension:

$$
\begin{aligned}
& \bar{f}=\frac{f c}{\rho V^{2}} \\
& \bar{n}=\frac{n c}{\rho V^{2}} \\
& \bar{T}=\frac{T}{\rho V^{2}}
\end{aligned}
$$

When we rewrite the equation of motion with these dimensionless quantities we obtain

$$
\bar{D}^{2} \bar{r}+2 \Omega \times \bar{D} \bar{r}+\Omega \times(\Omega \times \bar{r})+\Omega \frac{\partial \Omega}{\partial \bar{t}} \times \bar{r}=\frac{\partial}{\partial \bar{s}}\left(\bar{T} \frac{\partial \bar{r}}{\partial \bar{s}}\right)+f
$$

Only one parameter remains in this equation (without taking into account the external force term). It is the dimensionless angular velocity $\Omega$ :

$$
\Omega=\frac{c \omega}{V}
$$

This is the single most important parameter in our model. We will show later on that we can make many important conclusions if we determine how $\Omega$ changes with time as the yarn is being unwound.

There are two additional dimensionless parameters: $\mu$, the coefficient of friction between yarn and package, and the coefficient of air resistance $\mathrm{p}_{0}$ :

$$
p_{0}=\frac{16 c D_{n}}{\rho}
$$

These two parameters are approximately constant during the unwinding, so they are less important.The coefficient of friction depends on the quality of the yarn and on the surface properties of the package. The quality of the surface is comparable for forward and backward unwinding direction, so that the coefficient of friction remains approximately constant.

\section{SIMULATION MODEL}

On cylindrical packages the angular velocity depends od the winding angle $\phi$ according to relation:

$$
\omega=\frac{V}{C}\left(\frac{1}{\cos \phi}-\tan \phi\right)^{-1}=\frac{V}{C} \frac{\cos \phi}{1-\sin \phi}
$$

In deriving this expression we neglected the variation of yarn length in the balloon during the time interval when two layers unwind. 
The dimensionless angular velocity can obviously be expressed as:

$$
\Omega=\frac{\cos \phi}{1-\sin \phi}
$$

According to our simple model the dimensionless angular velocity thus only depends on the winding angle which will change with time because this angle is different for layers that are unwinding from front towards rear edge and those that are unwinding as the unwinding point moves from the rear towards front edge. The dependance of $\Omega$ on the winding angle is shown in Fig. 2.

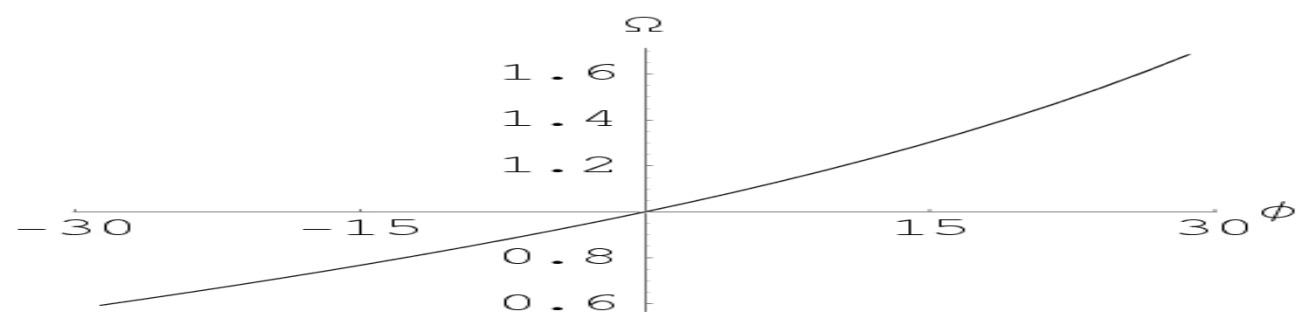

Fig.2 Dependence of $\Omega$ on the winding angle [9]

In conical packages the relation is only slightly modified:

$$
\omega=\frac{V}{c} \frac{\cos \phi}{1-\cos \alpha \sin \phi}
$$

Here $\alpha$ is the apex angle of the conical package. For typical values of $\alpha$ there is little difference between cylindrical and conical packages.

During unwinding the lift-off point moves up and down the package. We can presume that the winding angle is approximately constant in the middle of the package and it changes at the edges of the package where its sign is reversed. To describe the time dependence of the winding angle we must look for a periodic function, because motion of the point is periodic to a good approximation. The most known periodic functions are trigonometric function, such as sine function. This function should be modified so that it will change only slightly when the point moves up or down the packages. We can achieve this by raising the sine to a low fractional power, say $1 / 40$ (we have to be careful about the signs, so we take absolute value of sine function and restore the sign using the signum function:

$$
f(t)=\operatorname{sign}(\sin t)|\sin t|^{\frac{1}{40}}
$$

The diagram of this function is shown in fig.3.

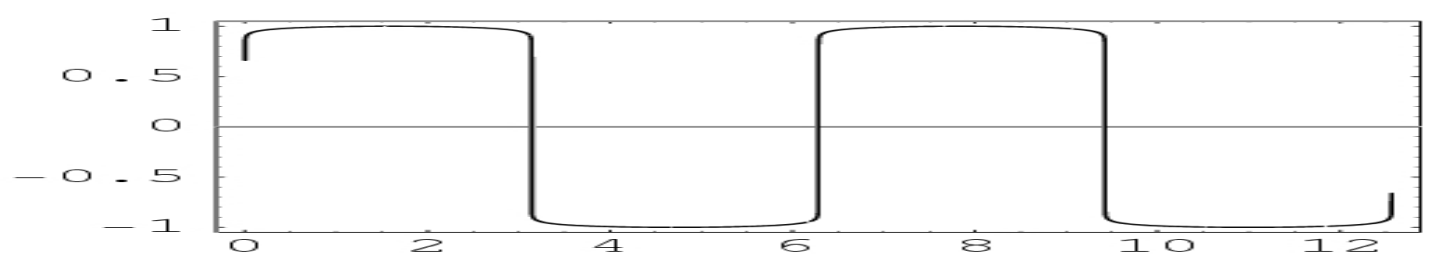

Fig. 3 Model function for winding angle 
The physical reality is somewhat different [10], especially in cross-wound packages, made using circumferential driving of the tube. The diagram shown in Fig. 3 would apply to precision wound cross-wound packages, made using direct driving of the tube. The loops would then lie next to each other.

If we considered a few loops of the rear end of the tube, before the next layer starts to unwind in the direction of the front end, and a few loops from the front end of the tube, before the next layer begins to unwind in the direction of the rear end, then we would obtain the diagram in Fig. 3. In this case we would have to neglect all the loops in between.

Speaking of the unwinding process, we are mostly interested in the maximal tensions in the yarn and the oscillations of the tension as a function of the unwinding speed. We aim to achieve the highest possible speed, while keeping the tension in the yarn and oscillations as low as possible.

The variations of the dimensionless angular velocity (Fig. 4) are more pronounced in cross wound packages with a high winding angle. In cross-wound packages it is therefore inadmissible to consider the winding angle as a small quantity and to neglect it in the first approximation

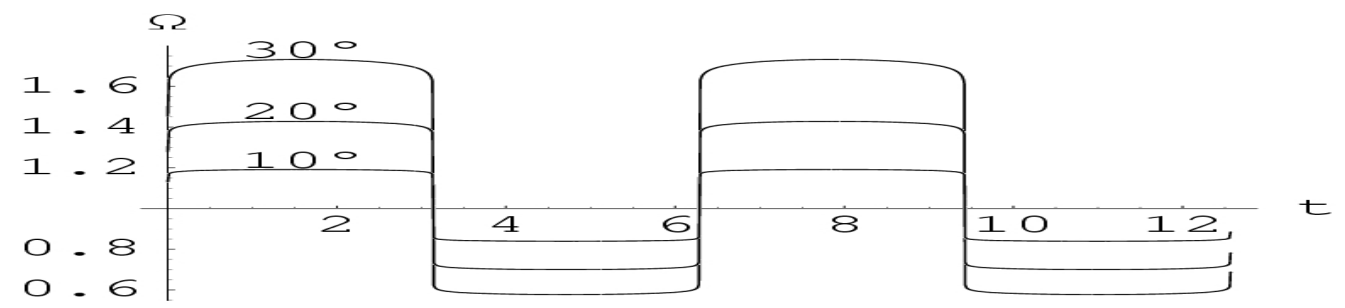

Fig. 4 Variations of the dimensionless angular velocity

The lower the winding angle is, the lower will the oscillations of the tension be. The maxima will then be lower and we'll be able to increase the unwinding speed. The problem is that most of the winding machines in use throughout the world use circumferential driving of the tube. This method of package winding makes it impossible to change the winding angle, while this is possible with winding machines using direct driving of the tube. In recent times the fraction of such winding machines is increasing.

\section{CONCLUSION}

Cross-wound packages made using circumferential driving of the tube do not permit to achieve the unwinding speeds necessary on fast weaving loom, where the cross-wound package is used as a wefting package. For this reason two wefting packages are necessary for every color of the weft, as well as two weft feeders of weft which is expensive and irrational.

In circumferential driving of the package the winding angle does not change. The direct consequence is the mirror winding - the winding of one layer on top of the other, which can be avoided by modulation. The threads do not lie parallel to each other (the pitch of the helix is usually large and the points of contact of two consecutive layers are 
sparse). Because of the large contact area it occurs that entire layers slip or that the yarn breaks.

In modern winding machines with a direct driving of the tube, the winding angle can be changed. Each layer has a determined winding angle, so that there are more points of contact as in the packages made with circumferential driving of the tube. Of special importance is the fact, that loops on one layer aren't parallel to the loops in the next layer, which reduces the possibility of slips during unwinding. Furthermore there are no difficulties of mirror winding, so that there is no danger of yarn falling off the package.

The winding machines with a direct driving of the tube allow greater flexibility of winding of cross-wound packages compared to the winding machines with a circumferential driving. They allow construction of packages that can be unwound at higher unwinding speeds.

In no case can we avoid the oscillations of yarn tension near the edges of a package, when the direction of motion of unwinding and lift-off points changes. The winding of the packages has to be optimized so that the absolute value of the yarn oscillation is as low as possible.

\section{REFERENCES}

1. A. E. D. Barr, H. Catling. Manual of Cotton Spimmimg, Volume Five. Butterworth, 1976.

2. D.G. Padfielf, A note on fluctuations of tension during unwinding, J.Text.Inst $\mathbf{4 7}$ 301-308, 1956.

3. C. Mack, Theoretical study of ring and cap spinning balloon curves(with and without air drag), J.Text.Inst 44, 483-498, 1953.

4. D. G. Padfield, The Motion and Tension of an Unwinding Thread. Proc. R. Soc., vol. A245, 382-407, 1958.

5. V. K. Kothari, G. A. V. Leaf, The unwinding of yarns from packages, PartI: The theory of yarn-unwinding, J.Text.Inst 70 (3)89-95, 1979.

6. W. B. Fraser, T. K. Ghosh, S. K. Batra, On unwinding yarn from cylindrical package. Proc. $R$. Soc. Lond. A, 436, 479-498, 1992.

7. S. Praček, Modification of yarn unwinding dynamics. Dissertation, University of Ljubljana, 2002.

8. J. A. Roberson, C. T. Crowe, Engineering fluid dynamics. Houghton Mifflin Company, Boston, second edition, 1980.

9. X. M. Kong, Steady state unwinding of yarn from cylindrical packages: theory and experiment. Dissertation, Clemson University, 1997.

10. D. Jakšić, Priprava osnove in votka za tkanje. Tekstilec, NTF, Ljubljana, 2001. 